\title{
1 Melt electrowritten scaffolds with bone-inspired fibrous and mineral 2 architectures to enhance BMP2 delivery and human MSC osteogenesis
}

3 Kian F. Eichholz. ${ }^{1,2,3}$, David A. Hoey. ${ }^{1,2,3,4}$

$4{ }^{1}$ Dept. Mechanical, Aeronautical and Biomedical Engineering, Materials and Surface Science Institute, University of

5 Limerick, Limerick, Ireland

$6{ }^{2}$ Trinity Centre for Bioengineering, Trinity Biomedical Sciences In stitute, Trinity College Dublin, Ireland

$7{ }^{3}$ Dept. of Mechanical and Manufacturing Engineering, School of Engineering, Trinity College Dublin, Ireland

$8 \quad{ }^{4}$ Advanced Materials and Bioengineering Research Centre, Trinity College Dublin \& RCSI

\section{Abstract}

11 Material micro-architecture and chemistry play pivotal roles in driving cell behaviour. Bone at a 12 cellular level consists of arranged fibres with a cross-fibrillar mineral phase made up of curved nano13 sized needle shaped crystals. This nano-structured mineral architecture can bind and stabilise 14 proteins within bone for centuries and thus holds promise as a strategy for therapeutic delivery in 15 regenerative medicine. Herein, we use melt electrowriting (MEW) technology to create fibrous 3D 16 PCL micro-architectures. These scaffolds were further modified with an extrafibrillar coating of plate 17 shaped micron-sized calcium phosphate crystals $(\mathrm{pHA})$, or with a novel extrafibrillar coating of 18 needle shaped nano-sized crystals (nnHA). A third scaffold was developed whereby nano-sized 19 crystals were placed intrafibrillarly during the MEW process (iHA). X-ray diffraction revealed altered 20 crystal structure and crystallinity between groups, with hydroxyapatite (HA) being the primary phase 21 in all modifications. Water contact angle was investigated revealing increased hydrophilicity with 22 extrafibrillar coatings, while tensile testing revealed enhanced stiffness in scaffolds fabricated with 23 intrafibrillar HA. Biological characterisation demonstrated significantly enhanced human 24 stem/stromal cell mineralisation with extrafibrillar coatings, with a 5-fold increase in mineral 25 deposition with plate like structures and a 14-fold increase with a needle topography, demonstrating 26 the importance of bone mimetic architectures. Given the protein stabilising properties of mineral, 27 these materials were further functionalised with BMP2. Extrafibrillar coatings of nano-needles 28 facilitated a controlled release of BMP2 from the scaffold which further enhanced mineral 29 deposition by osteoprogenitors. This study thus outlines a method for fabricating scaffolds with 30 precise fibrous micro-architectures and bone mimetic nano-needle HA extrafibrillar coatings which 31 significantly enhance mesenchymal stem/stromal cell (MSC) osteogenesis and therapeutic delivery 32 and thus hold great promise for bone tissue regeneration. 


\section{Introduction}

34 Autografts are the gold standard for bone defect repair due to their osteoconductive, osteoinductive 35 and immunocompatible properties, but unfortunately are limited in supply [1]. Due to technology 36 advancements and an improved understanding of the physiological mechanisms of bone repair, 37 synthetic tissue engineering approaches have demonstrated great progress in developing 38 bioinspired materials that can promote bone formation [2]. These approaches continue to fall short 39 of the gold standard however, therefore, continued development is required. Bone is a complex, 40 hierarchical multi-phasic organ comprised primarily of mineralised collagen fibrils spanned by 41 additional continuous, cross-fibrillar mineral units $[3,4]$. It was only recently identified that the first 42 fundamental component in this hierarchy is composed of needle-shaped mineral units of base $5 \mathrm{~nm}$ 43 and length $50-100 \mathrm{~nm}$, which merge to form platelets and mineral stacks and aggregate to form 44 irregular 3D structures of size $200-300 \mathrm{~nm}$ which are crossfibrillar in nature [4]. It is the 45 combination of collagen and mineral with specific topographies, working in unison in this 46 hierarchical structure, which gives the bone both its excellent biological and mechanical properties.

47 Moreover, this mineral nano-topography identified in bone is believed to be a key contributor to non-collagenous protein binding and stabilisation, preserving proteins for centuries within the tissue without denaturation [5]. This therefore provides a potential strategy for therapeutic delivery in regenerative medicine [6]. Recapitulating this unique multiscale architecture may represent an

51 innovative approach to develop effective bioinspired materials to promote bone repair.

52 Bone formation occurs following mesenchymal stem/stromal cell osteogenic differentiation into an 53 osteoblast, which begins laying down collagen fibrils and enriching the surrounding environment 54 with alkaline phosphatase and osteocalcin to promote mineral deposition with in the collagen template $[7,8]$. The resulting nano-crystals are initially formed within collagen fibrils, eventually nucleating to fill the voids forming a composite structure of mineral and collagen fibres approximately $10 \mu \mathrm{m}$ in diameter [9]. The underlying collagen fibrillar template is thus crucial in dictating cellular behaviour in bone and many other tissues, with dysregulation of this unique structure leading to undesirable outcomes including cell death, abnormal differentiation and tumour development $[10,11]$. Through the advent of new technology such as 3D printing, precise control of scaffold architecture is now achievable [12]. Fused deposition modelling (FDM) can produce scaffolds with defined fibre architecture creating pores of differing shapes [13] in addition to facilitating mineral incorporation [14-18], which in turn drives mesenchymal stem/stromal cell (MSC) differentiation down specific lineages. However, FDM is limited in that fibre diameters below 100 
fibre diameters on the nano/micron scale which have been utilised in bone tissue engineering strategies [20, 21]. However, due to the unstable "whipping" motion of the electrospinning jet during fibre deposition, there is limited control over fibre architecture using this approach. Melt Electrospinning Writing (MEW) is a recently developed 3D printing technology which overcomes the above limitations of FDM and electrospinning, being capable of fabricating and controlling the deposition of micron-scale fibres [22-24]. This facilitates the manufacture of complex fibrous microarchitectures [25-27] consistent with that seen in native extracellular matrix (ECM) including that of bone. We recently utilised MEW to fabricate scaffolds with bone inspired fibre diameters of $10 \mu \mathrm{m}$ and investigated whether the fibre architecture in terms of degrees of alignment could influence stem/stromal cell behaviour [28]. Interestingly, the combination of fibre diameter and alignment had significant impact on cell shape, cell signalling and ultimately MSC osteogenesis, with a $90^{\circ}$ fibre architecture mediating a 4-fold increase in stem/stromal cell mineralization when compared to $10^{\circ}$ aligned architectures or a traditional random electrospun scaffold, demonstrating the critical role of fibrillar architecture in mediating stem/stromal cell behaviour and highlighting MEW as a powerful tool to fabricate physiological bioinspired environments for tissue engineering strategies.

In bone tissue, the mineral component makes up $60-70 \%$ of its dry weight, and is composed primarily of non-stoichiometric apatite along with other loosely bound ions including $\mathrm{Ca}^{2+}, \mathrm{HPO}_{4}{ }^{2-}$, $\mathrm{CO}_{3}{ }^{2-}$ and $\mathrm{Mg}^{2+}$ in a hydrated layer at the surface [29]. As with fibrillar architecture, the mineral structure in bone plays a key role in mediating cell behaviour, with deficiencies or changes in mineral composition contributing to diseases such as osteoporosis [30]. This has been exploited in a host of strategies for orthopaedic and dental applications, where materials and surfaces have been modified with calcium phosphates with the aim of creating biomimetic materials which promote osseointegration and tissue regeneration. This includes coatings for implant fixation, cements and adhesives for orthopaedic and dental implants, and synthetic bone grafts [31]. A wide range of commercially available materials have been investigated, including hydroxyapatite (HA), calcium deficient apatite (CDA), $\alpha$ - and $\beta$ tricalcium phosphate (TCP) and biphasic calcium phosphates such as HA + TCP, with a range of other materials including dicalcium phosphate (DCP) and Tetracalcium phosphate (TTCP) also being investigated [32]. As with fibrillar architecture, an ever increasing appreciation for the importance of the architecture and particle size of the mineral phase is being gained, which defines surface topography and thus has a considerable influence on cell behaviour and scaffold performance [33]. When cultured on nano-scale $\mathrm{HA}(\mathrm{nHA})$ films with varying particles sizes from $20-80 \mathrm{~nm}$ in diameter, MSCs exhibit a trend of greater viability and proliferation with smaller particle sizes [34]. The osteogenic capacity of $\mathrm{nHA}$ has been further demonstrated in a range of studies where cells have been cultured in nHA suspensions with particles of size between $20-100$ 
$101 \mathrm{~nm}$, with cells demonstrating uptake of particles and exhibiting enhanced osteogenic differentiation

102 [35-37]. The role of HA particle size has also been investigated in the 3D printing of composite PCL-

103 HA blends, with enhanced proliferation and alkaline phosphatase (ALP) activity of MSCs seen with 104 nano-particles compared to micro-particles [38]. Remarkably, particle size has not only been shown 105 to be an important cue for MSC osteogenesis, but also plays a role in suppressing undesired cellular 106 outcomes in bone. This has been demonstrated via osteosarcoma inhibition [34] and reduced melanoma proliferation [39] with nHA compared to larger particles, further reinforcing the therapeutic potential of nano-scale particles for additional applications such as bone regeneration following tumour ablation. A recent study has also demonstrated the regenerative capacity of grafts with a needle surface topography in-vivo, with sub-micron needles triggering earlier and accelerated bone formation [40].

Bone tissue also houses non-collagenous proteins and growth factors embedded within its composite matrix, which are remarkably resolute and can be preserved for centuries due to the great stability provided by the mineral structure [5]. It is thus of no surprise that the great binding affinity to HA of a host of biological and synthetic components has been exploited for therapeutic benefits. HA has been extensively used as a delivery mechanism for BMP2 protein with proven success in a number of in-vivo models [41-43], while nHA particles have also been used for non-viral gene delivery of BMP2 to enhance MSC osteogenesis [44, 45]. Anti-tumour drugs have also been combined with $\mathrm{nHA}$ as a delivery vector, with potential applications in osteosarcoma chemotherapy [46] and the development of targeted drug delivery methods [47]. Interestingly, protein binding and stabilization is closely related to the nanoscale structural features of the mineral coatings [6], demonstrating the importance of controlling this $\mathrm{nHA}$ architecture. In addition to the already proosteogenic capabilities of biomimetic nHA, there is great potential to exploit its natural affinity to further advance treatments by facilitating therapeutic delivery providing an extensive range of enhanced regenerative benefits.

In this study, we aim to recapitulate the native structure of bone via the development of a biomimetic tissue regeneration strategy comprised of a micro-fibrous matrix spanned by an intrafibrillar nano-needle mineral phase stabilising bound protein for enhanced tissue regeneration. Herein, we use melt electrowriting (MEW) technology to create fibrous 3D PCL micro-architectures. These scaffolds were further modified with an extrafibrillar coating of plate shaped micron-sized calcium phosphate crystals $(\mathrm{pHA})$, or with a novel extrafibrillar coating of needle shaped nano-sized crystals (nnHA). A third scaffold was developed whereby nano-sized crystals were placed intrafibrillarly during the MEW process (iHA). Along with vastly differing surface topographies, 
134

135

136

137

138

139

140

141

142

143

144

145

146

147

148

surface chemistry was also altered, with calcium/phosphate ratios within the range seen in healthy native bone. Biological characterisation demonstrated significantly enhanced human stem/stromal cell mineralisation with extrafibrillar coatings, with a needle topography producing the most robust bone formation, demonstrating the importance of bone mimetic architectures. Given the protein stabilising properties of mineral, these materials were further functionalised with BMP2. Extrafibrillar coatings of nano-needles facilitated a controlled release of BMP2 from the scaffold which further enhanced mineral deposition by osteoprogenitors. This study thus outlines a method for fabricating scaffolds with precise fibrous micro-architectures and bone mimetic nano-needle HA extrafibrillar coatings which significantly enhance MSC osteogenesis and therapeutic delivery and thus holds great promise for bone tissue regeneration.

\section{Materials and methods}

\subsection{Scaffold fabrication}

\subsubsection{Polycaprolactone (PCL) melt electrowriting}

Fibrous scaffolds with a fibre diameter of $10 \mu \mathrm{m}$, square apparent pore size of $50 \mu \mathrm{m}$, and layer offset of 300[? $\mu \mathrm{m}$ were fabricated on a custom built MEW apparatus as previously described [28]. We have demonstrated that this fibrous scaffold architecture is optimal for MSC osteogenesis [28]. Briefly, heated air at a temperature of $90^{\circ} \mathrm{C}$ was circulated around a syringe to melt polycaprolactone (PCL) (Sigma Aldrich 440744, average Mn 80,000), with air pressure being used to extrude the polymer through a $21 \mathrm{G}$ needle with high voltage applied at a distance of $15 \mathrm{~mm}$ from a grounded aluminium collector plate. Fibres were deposited with a $90^{\circ}$ offset between subsequent layers to result in a square pore shape. Scaffolds for control (C) groups were used without further mineral modification.

\subsubsection{Plate hydroxyapatite $(\mathrm{pHA})$ coating}

This coating was carried out via a saturated simulated body fluid (SBF) solution, as described by Martine et al [48]. Reactions were carried out in $50 \mathrm{ml}$ conical tubes, with reagent volumes being maintained at $40 \mathrm{ml}$ at each step of the process and 6 scaffolds of dimension $3 \times 3 \mathrm{~cm}$ being processed per tube. Scaffolds were immersed in $70 \%$ ethanol for 15 min under vacuum and treated with $2 \mathrm{M} \mathrm{NaOH}$ for $45 \mathrm{~min}$ at $37^{\circ} \mathrm{C}$ following a $5 \mathrm{~min}$ vacuum treatment to remove air bubbles. Scaffolds were rinsed 5 times in MilliQ water and immersed in 10x SBF, previously brought to a $\mathrm{pH}$ of 6 for $30 \mathrm{~min}$ at $37^{\circ} \mathrm{C}$ following a 5 min vacuum treatment. The coating step in $10 \times$ SBF was repeated a further two times minus the vacuum treatment. Scaffolds were then treated with $0.5 \mathrm{M} \mathrm{NaOH}$ for 
$16530 \mathrm{~min}$ at $37^{\circ} \mathrm{C}$, rinsed 5 times in MilliQ water and allowed to dry overnight. Scaffolds treated with

166 this coating method are defined as plate hydroxyapatite (pHA).

\section{$167 \quad 3.1 .3 \quad$ Nano-needle hydroxyapatite ( $\mathrm{nnHA}$ ) coating}

168 We developed a nano-hydroxyapatite coating to coat scaffolds with nano-needle mineral units.

169 Sample pre- and post-processing was carried out as per the calcium phosphate coating procedure,

170 with the 10x SBF treatment step being substituted for alternative calcium and phosphate solutions

$171[49,50]$. The calcium solution was made with $0.05 \mathrm{M}$ calcium chloride dihydrate (Sigma Aldrich

172 C7902) in MilliQ water. The phosphate solution was made with $0.03 \mathrm{M}$ sodium phosphate tribasic

173 dodecahydrate (Sigma Aldrich S7778) and 0.01M sodium hydroxide in Millia water. The entire

174 coating procedure is outlined as below. Scaffolds were immersed in $70 \%$ ethanol for 15 min under

175 vacuum and treated with $2 \mathrm{M} \mathrm{NaOH}$ for $45 \mathrm{~min}$ at $37^{\circ} \mathrm{C}$ following a $5 \mathrm{~min}$ vacuum treatment to

176 remove air bubbles. Scaffolds were rinsed 5 times in MilliQ water and added to $20 \mathrm{ml}$ of the calcium

177 solution. $20 \mathrm{ml}$ of the phosphate solution was slowly added to the calcium solution, and scaffolds

178 were incubated for $30 \mathrm{~min}$ at $37^{\circ} \mathrm{C}$ following a $5 \mathrm{~min}$ vacuum treatment. This coating procedure was

179 repeated a further two times minus the vacuum treatment. Scaffolds were then treated with $0.5 \mathrm{M}$

$180 \mathrm{NaOH}$ for $30 \mathrm{~min}$ at $37^{\circ} \mathrm{C}$, rinsed 5 times in MilliQ water and allowed to dry overnight. Scaffolds

181 treated with this coating method are defined as nano-needle hydroxyapatite (nnHA).

\section{3.1.4 Hydroxyapatite incorporated (iHA) PCL melt electrowriting}

183 A nHA-PCL blend was created by incorporating a 3.5wt\% of nano-hydroxyapatite (Sigma Aldrich 184 677418) into the polymer. PCL and nano-hydroxyapatite with a total mass of $1 \mathrm{~g}$ was added to a 185 glass vial, with $10 \mathrm{ml}$ of chloroform being added under stirring until dissolved. Particles were 186 dispersed in the solution by sonication for $1 \mathrm{~h}$, after which it was poured into a glass dish and left 187 overnight for chloroform to evaporate. The resulting film was formed into pellets which were loaded 188 into $1 \mathrm{ml} \mathrm{BD}$-Braun syringes. The syringes were heated for $1 \mathrm{~h}$ at $110^{\circ} \mathrm{C}$, and centrifuged at $4,000 \mathrm{~g}$ 189 for $60 \mathrm{~s}$ to remove air. The previous heating and centrifugation steps were repeated for a total of 8 190 cycles, or until a homogenous blend was present in the syringe with no air bubbles. MEW processing 191 parameters were optimised to facilitate controlled deposition of PCL-nHA blends, with temperature 192 increased to $110^{\circ} \mathrm{C}$ and voltage increased by approximately $0.5 \mathrm{kV}$. Scaffolds for this modification 193 method are defined as fibre incorporated hydroxyapatite (iHA). 


\section{$194 \quad 3.2$ Scaffold characterisation}

\section{3.2.1 SEM imaging and Energy-dispersive X-ray spectroscopy}

196 For SEM imaging, samples were prepared for imaging by coating with gold/palladium for $40 \mathrm{~s}$ at a 197 current of $40 \mathrm{~mA}$. For analysis with energy-dispersive X-ray spectroscopy (EDX), scaffolds were

198

199

200

201

202

203

204

205

206

207

208

209

210

211

212

213

214

215

216

217

218

219

220

221

222

223

224 carbon coated and analysed at a voltage of $15 \mathrm{kV}$ in a Zeiss ULTRA plus SEM with an $80 \mathrm{~mm}^{2}$ Oxford Inca EDX detector. To investigate approximate calcium/phosphorus atomic ratios for each group, spectra were acquired on scaffolds for $30 \mathrm{~s}$ ( $n=6,2$ technical replicates per scaffold). Element maps were constructed by taking 2 frames at a resolution of $256 \times 192$ with map dwell of $4000 \mu \mathrm{s}$ and linescan dwell of $2000 \mu \mathrm{s}$.

\subsubsection{X-ray diffraction}

To obtain powder samples for x-ray diffraction (XRD) analysis, $10 \times$ SBF was allowed to precipitate in solution at a $\mathrm{pH}$ of 6 , while for the nnHA group, calcium and phosphate solutions were mixed together and allowed to precipitate. Solutions were centrifuged at 5,000 g for $40 \mathrm{~min}$ to collect the precipitate, which was then placed in dishes and dried for $4 \mathrm{~h}$ at $50^{\circ} \mathrm{C}$ in an oven. The resulting mineral was ground into a powder using a mortar and pestle. The hydroxyapatite powder used for iHA was used for XRD analysis without any further processing. Samples were loaded in a Brucker D8 ADVANCE powder diffractometer and run for $1 \mathrm{~h}$ from $5-80^{\circ}$ in the $2 \theta$ range with a monochromatic $\mathrm{Cu}-\mathrm{K} \alpha$ radiation source. Values for crystallinity and crystallite size were calculated using DIFFRAC.SUITE EVA 4.1.1. Crystallite size was calculated from taking 5 measurements of the (211) peak in pHA and iHA groups. In the iHA group, crystallite size was calculated from the peak in the position of (211), (112) and (300), due to the broad peaks resulting in their merging.

\subsubsection{Calcium staining}

Samples were taken from scaffolds using a $2 \mathrm{~mm}$ biopsy punch $(n=5)$ and incubated with $1 \%$ alizarin red s (Sigma Aldrich, A5533) in distilled water for $10 \mathrm{~min}$ at a pH of between $4.1-4.3$. Samples were rinsed 3 times with water and allowed to dry prior to imaging. To quantify bound stain, $400 \mu \mathrm{l}$ of $10 \%$ acetic acid was applied and samples incubated at room temperature for $30 \mathrm{~min}$ while shaking at $150 \mathrm{rpm}$. The acetic acid was added to $1.5 \mathrm{ml}$ tubes, vortexed vigorously and heated to $85^{\circ} \mathrm{C}$ for 10 $\mathrm{min}$. Tubes were transferred to ice for $5 \mathrm{~min}$, centrifuged at 20,000 g for $15 \mathrm{~min}$, and $300 \mu \mathrm{l}$ of the supernatant was added to new tubes along with $120 \mu \mathrm{l}$ of $10 \%$ ammonium hydroxide. Standards were made with dilutions of alizarin red solution in distilled water, with the $\mathrm{pH}$ of each adjusted to between $4.1-4.3$. Samples and standards were read at $405 \mathrm{~nm}$ in a 96-well plate. 


\section{$225 \quad 3.2 .4$ Water contact angle}

226 The water contact angle of all scaffold groups was quantified using a FTA125 contact angle analyzer 227 (First Ten Angstroms Inc.). Samples were prepared using an $8 \mathrm{~mm}$ biopsy punch ( $\mathrm{n}=6-7)$. All measurements were taken 10 frames after contact of the water with the sample.

\subsubsection{Tensile testing}

Rectangular samples $(n=4)$ of dimension $5 \times 20 \mathrm{~mm}$ were taken from scaffolds and used for tensile testing in a Zwick Z005 with 5? load cell (A.S.T. - Angewandte System Technik GmbH). Samples were pre-loaded to 0.002 ? $\mathrm{N}$ at a speed of 1 ? $\mathrm{mm} / \mathrm{min}$, and then loaded at a speed of 10 ? $\mathrm{mm} / \mathrm{min}$ with a time save interval of 0.1 ? $\mathrm{l}$ s. Load-displacement curves were graphed and used to determine the stiffness, toe region stiffness and yield force for each group.

\subsection{Human bone marrow mesenchymal stem/stromal (hMSC) cell culture}

Scaffolds were punched to a diameter of $8 \mathrm{~mm}$ and UV sterilised for $20 \mathrm{~min}$ on each side before being mounted in stainless steel holders and placed in 24-well plates. Scaffolds were then pre-wet in a graded ethanol series of $100 \%, 90 \%$ and $70 \%$ for 20 min each before being washed three times in phosphate buffered saline (PBS). They were then incubated overnight in DMEM with $10 \%$ FBS at $37^{\circ} \mathrm{C}$. Human bone marrow mesenchymal stem/stromal cells (hMSCs) were isolated from bone marrow (Lonza, US), trilineage potential verified (data not shown), and seeded at a number of 10,000 cells per scaffold. Scaffolds were transferred to new well plates after $24 \mathrm{~h}$, and cultured in osteogenic medium (OM) from day 3, which consisted of 10\% FBS DMEM supplemented with 100 ? $\mathrm{nM}$ dexamethasone, 10 [? $\mathrm{mM} \beta$-glycerol phosphate and 50 ? $\mathrm{\mu g} / \mathrm{ml}$ ascorbic acid. Medium was changed every 3.5 days.

\subsection{Proliferation}

247 At days $1,7,14$ and 21 , scaffolds were added to 100 ? $] \mu$ lysis buffer $(n=4)$ containing $0.2 \%$ Triton $X$ 248 100,1] $\mathrm{mM}$ Tris $\mathrm{pH}$, with phenylmethylsulfonyl fluoride (PMSF) being added at a ratio of 1:200 just 249 before use, and stored at $-80^{\circ} \mathrm{C}$. Before DNA quantification, samples underwent sonication for $60 \mathrm{~s}$ 250 and subjected to three freeze-thaw cycles in liquid nitrogen before being stored on ice. DNA content 251 was quantified using a Quant-iT ${ }^{\mathrm{TM}}$ PicoGreen $^{\text {TM }}$ dsDNA Assay Kit (Invitrogen, P7589), with excitation 252 and emission wavelengths of 485 ? $\mathrm{nm}$ and 528 ? $\mathrm{nm}$ respectively. The DNA content in 10,000 hSSCs 253 seeded and pelleted in centrifuge tubes was also quantified to calculate the total number of cells 254 present on scaffolds. 


\subsection{Characterisation of hMSC osteogenic differentiation}

\section{$256 \quad 3.5 .1$ Intracellular alkaline phosphatase (ALP)}

257 Intracellular ALP was quantified at days 14 and $21(n=4) .50 \mu$ l of $5 \mathrm{mM}$ pNPP was added to wells 258 along with $10 \mu \mathrm{l}$ of cell lysate and $70 \mu \mathrm{l} \mathrm{MilliQ}$ water in a 96-well plate. Standards were comprised of 259 serial dilutions of $p$-Nitrophenyl phosphate (pNPP, Sigma Aldrich, N1891) with $10 \mu$ l of $43 \mu \mathrm{M}$ ALP 260 enzyme (Sigma Aldrich, P6774) being added to each. Plates were incubated for $1 \mathrm{~h}$ in the dark at room temperature, and reactions were then stopped using $20 \mu \mathrm{l}$ of $3 \mathrm{M} \mathrm{NaOH}$. Absorbance was read on plates at $405 \mathrm{~nm}$, and ALP activity was calculated as the amount of pNPP generated as a function of sample volume and reaction time.

\subsubsection{Collagen production}

Scaffolds were cultured up to 21 days before evaluating collagen content. Cell-scaffold constructs were rinsed in PBS, fixed in 10\% neutral buffered formalin for $15 \mathrm{~min}$ and rinsed again in PBS before storage at $-20^{\circ} \mathrm{C}$. Scaffolds were stained with $200 \mu \mathrm{l}$ of $1 \mathrm{mg} / \mathrm{ml}$ of Direct Red 80 (Sigma Aldrich, 365548 ) in a saturated aqueous picric acid solution for $1 \mathrm{~h}$ with shaking at $150 \mathrm{rpm}$. Scaffolds were then washed twice with $0.5 \%$ acetic acid and allowed to dry overnight before imaging. To quantify collagen content, $500 \mu \mathrm{l} 0.5 \mathrm{M} \mathrm{NaOH}$ was added to wells under shaking until stain was dissolved, and solutions were added to $1.5 \mathrm{ml}$ tubes. Tubes were centrifuged at $14,000 \mathrm{~g}$ for $10 \mathrm{~min}$ to pellet debris. Standards were made by adding direct red staining solution to $8 \mu$ l of collagen I (Corning, \#354249) before centrifuging at $14,000 \mathrm{~g}$ for $10 \mathrm{~min}$ and re-suspending the collagen in $500 \mu \mathrm{l} 0.5 \mathrm{M} \mathrm{NaOH}$. The absorbance of samples and standards were read at $490 \mathrm{~nm}$ in 96 -well plates.

\subsubsection{Calcium production}

276 Cell-scaffold constructs were investigated for total calcium content after 21 days using $1 \%$ alizarin red S solution as previously described for calcium staining in scaffold characterisation. Cell-free scaffolds were also cultured up to 21 days to determine the contribution of total calcium from cell mineralisation versus the calcium present due to mineral modification of scaffolds.

\section{$280 \quad 3.6 \quad$ BMP2 functionalisation of MEW scaffolds}

\subsubsection{BMP2 adsorption onto scaffolds}

Scaffolds were prepared, sterilised and pre-wet in a graded ethanol series as previously described in section 3.3 before being washed three times in PBS. Recombinant human BMP2 (Peprotech 120-02) was diluted to $50 \mu \mathrm{g} / \mathrm{ml}$ in PBS, with $20 \mu \mathrm{l}$ of solution containing a total of $1 \mu \mathrm{g}$ BMP2 being placed 
285

286

287

288

289

290

291

292

293

294

295

296

297

298

299

300

301

302

303

304

305

306

307

308

309

310

on each scaffold and incubated for $4 \mathrm{~h}$ at room temperature. BMP2 was then removed and scaffolds were allowed to dry overnight.

\subsubsection{BMP2 release kinetics}

Scaffolds were placed in 48-well plates $(n=4)$ and $200 \mu \mathrm{l}$ of PBS added to each. At each time-point, PBS was removed and 1\% BSA added to result in a final BSA concentration of $0.1 \% .200 \mu \mathrm{l}$ of fresh PBS was then added to each well. Samples were stored at $-80^{\circ} \mathrm{C}$ until BMP2 quantity was determined via ELISA (R\&D Systems DY355-05). Cumulative BMP2 release was studied at time-points $1 \mathrm{~h}, 6 \mathrm{~h}$, and days $1,3,7,14$ and 21.

\subsubsection{Characterisation of osteogenic differentiation}

After BMP2 adsorption, 10,000 hMSCs were seeded onto scaffolds ( $n=4)$, and cultured up to 21 days after which DNA content was investigated to determine total cell number. Scaffolds were also cultured up to 21 days to assess osteogenic potential in terms of ALP activity, collagen production and calcium production as described previously.

\subsection{Statistical analysis}

Tensile testing and material characterisation data is presented in terms of average and standard deviation. Subsequent biological data is presented in terms of average and standard error of the mean. Statistical analysis was performed using one-way ANOVA and Tukey's multiple comparison post-test. Data from the BMP2 loading study was analysed using two-way ANOVA and Bonferroni's multiple comparison test.

\section{Results}

\subsection{MEW fibre topography is significantly altered by bulk and surface modification}

The topography of $90^{\circ}$ orientate fibrous MEW scaffolds was investigated following modification with calcium phosphate based materials, where significant changes were identified with minimal influence on macroscopic scaffold morphology. Control fibres have a diameter of $9.1 \mu \mathrm{m}(\mathrm{SD}=1.0$ ), with relatively featureless surface topography (Figure 1A). The pHA coating protocol yields a rosettelike arrangement of plates of average diameter $555.3 \mathrm{~nm}(S D=193.2)$ and thickness $26.0 \mathrm{~nm}$ (SD = 8.6) (Figure 1B). Total fibre diameter including the coating is $13.5 \mu \mathrm{m}$ ( $\mathrm{SD}=2.6$ ), giving an average coating thickness in the pHA group of $2.2 \mu \mathrm{m}$. In stark contrast to this, the nnHA coating protocol results in the formation of nano-needles on the fibre surface (Figure $1 \mathrm{C}$ ), with a length and diameter of $100.0 \mathrm{~nm}$ ( $S D=29.0$ ) and $37.0 \mathrm{~nm}(S D=7.1$ ) respectively. These needles form occasional aggregates which range in diameter from $150-500 \mathrm{~nm}$. Total fibre diameter shows a minimal 
316 increase compared to the control with a value of to $10.2 \mu \mathrm{m}$ (SD $=1.0$ ), yielding a total coating

317 thickness in this group of $0.55 \mu \mathrm{m}$. The incorporation of HA within fibres in iHA during the MEW

318 process yields minimal changes in surface topography, with the majority of visible particles being

319 seen as bulges just below the fibre surface, while several can also be seen to protrude above the

320 surface with no covering of PCL (Figure 1D). Particles have an average diameter of $147.2 \mathrm{~nm}$ (SD =

321 78.5), as measured within PCL fibres. The incorporation of HA also has an influence on the quality of

322 the MEW process, with periodic pulsing of the Taylor cone resulting in a larger fibre diameter

323 distribution (fibre diameter $=11.8 \mu \mathrm{m}, \mathrm{SD}=4.2$ ).

324
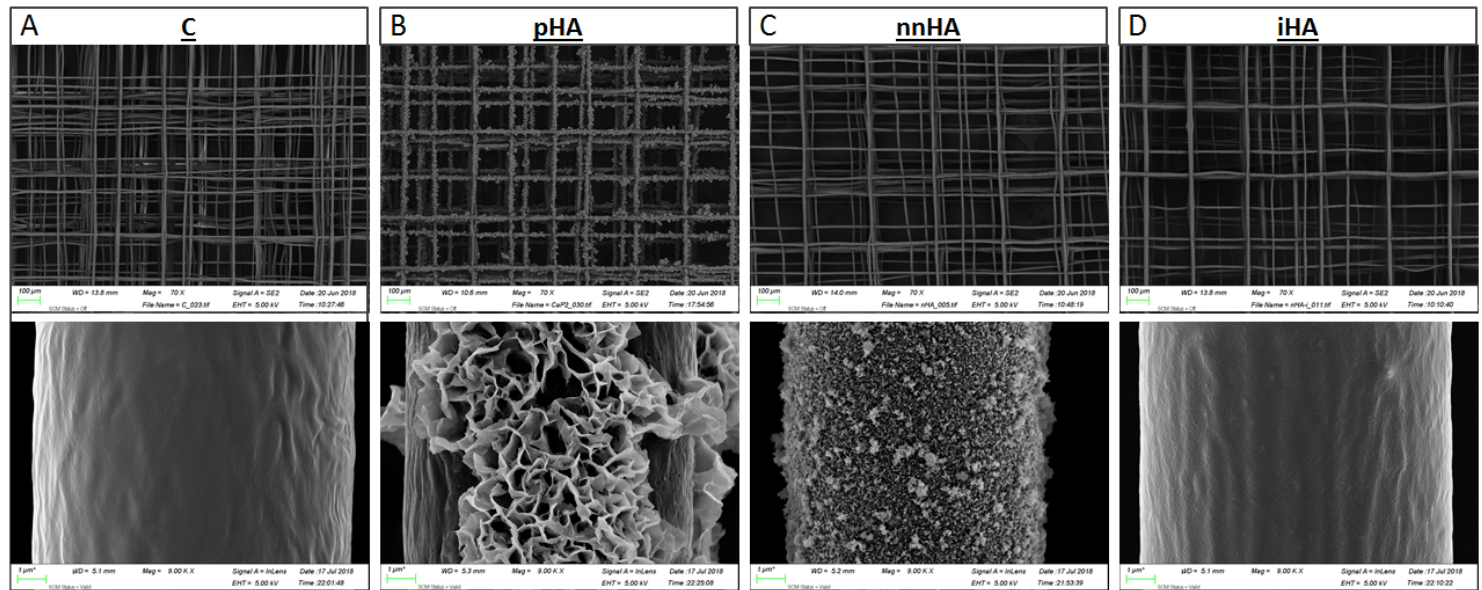

325
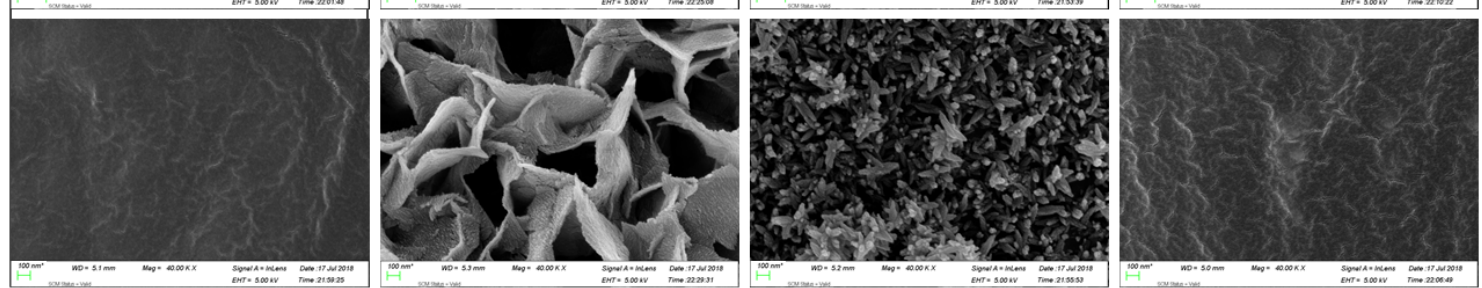

Figure 1 SEM imaging of scaffold groups illustrating the "plate" coating morphology in pHA, "needle" morphology in nnHA, and the presence of HA nanoparticles both within and protruding above the surface of the fibres in iHA.

\subsection{CaP treatments modify surface chemistry}

\subsubsection{Element analysis}

330 The coating chemistry and distribution in fibres was investigated via EDX analysis. The presence and

331 distribution of calcium and phosphorous was first investigated via element mapping where, as

332 expected, there was no detection in the control (Figure 2A). A consistent distribution of both 333 elements was seen in pHA and nnHA coated fibres, while a sparse distribution in the iHA group 334 confirmed the presence of HA particles within fibres. Carbon and oxygen were present in all groups 335 from the PCL, as well as calcium and phosphorous as seen in the spectra of all groups except the 
bioRxiv preprint doi: https://doi.org/10.1101/734855; this version posted August 13, 2019. The copyright holder for this preprint (which was not certified by peer review) is the author/funder, who has granted bioRxiv a license to display the preprint in perpetuity. It is made available under aCC-BY-NC-ND 4.0 International license.

336 control (Figure 2C, merge Figure 2D). A key difference in the pHA group was seen with the presence 337 of sodium and magnesium in this coating. $\mathrm{Ca} / \mathrm{P}$ ratios were investigated (Figure 2D) and were found 338 to be close to stoichiometric hydroxyapatite with a ratio of 1.66 for the pHA group. The iHA group 339 was investigated using both MEW scaffolds with HA incorporated, as well as the stock powder, with 340 both having a resulting ratio of 1.76 . The nnHA coating group deviated slightly from these findings, 341 with a more calcium-rich $\mathrm{HA}$ with a ratio of 1.97 being identified.
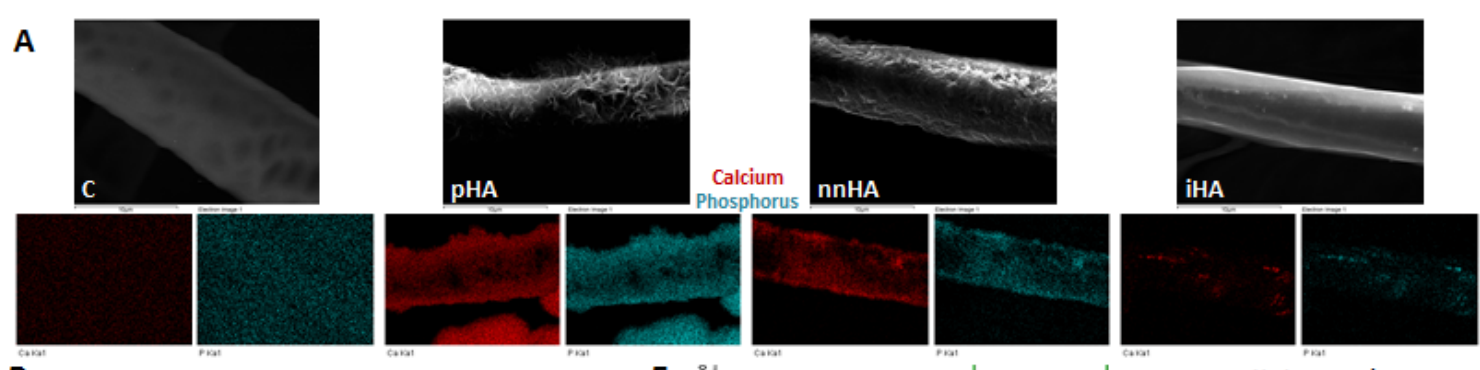

B
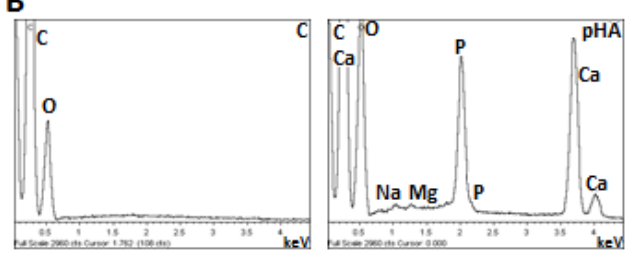

E

- Hydroxyapatite
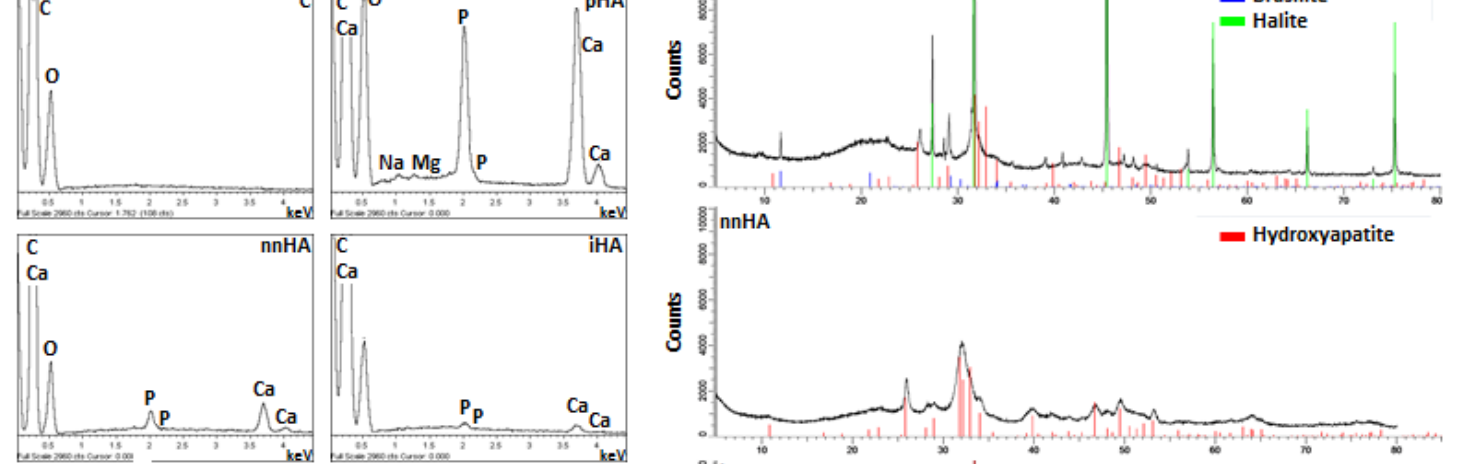

C

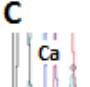

$\left\{\begin{array}{l}\text { Ca } \\ \text { lh }\end{array}\right.$

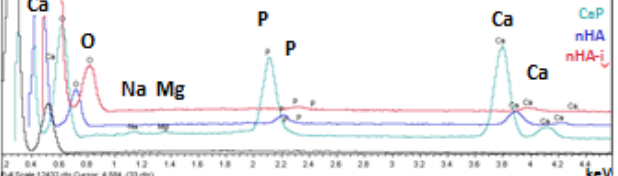

D
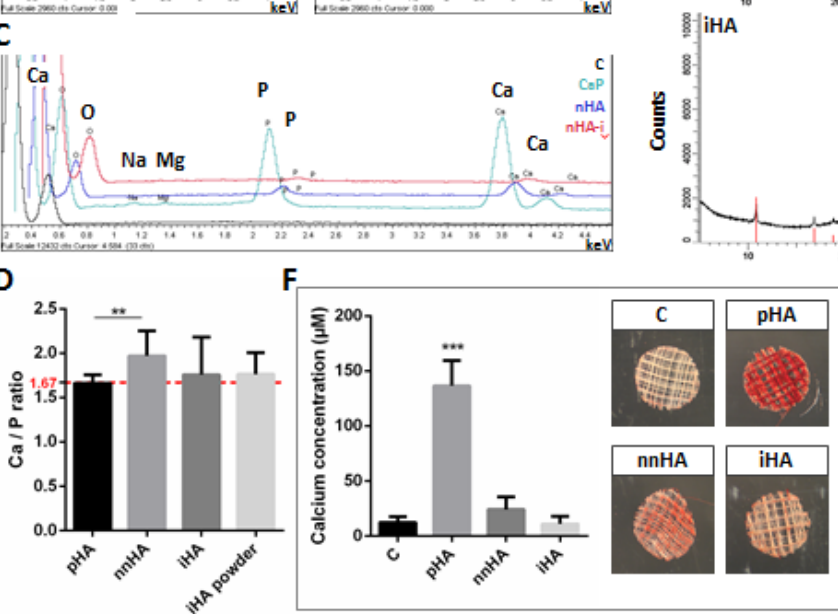

- Hydroxyapatite
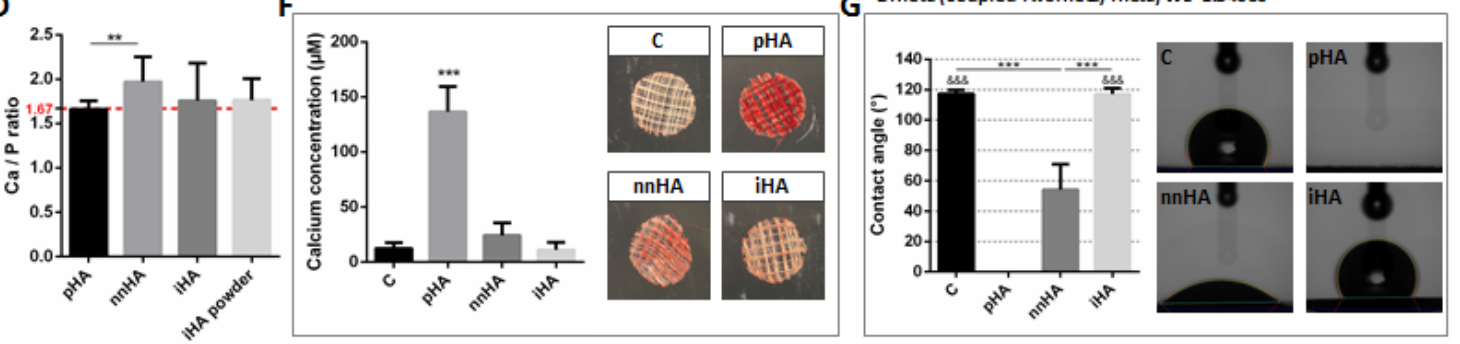

Figure 2 Chemical and physical characterisation of MEW-hydroxyapatite scaffolds. Element mapping of SEM images via EDX analysis illustrating the distribution of calcium and phosphorus in each group (A). Representative EDX spectra of all groups (B) and merge of spectra (C). Calcium to phosphorus ratios of EDX spectra ( $n=6$ scaffolds, $n=2$ technical replicates) (D). XRD analysis of powder representative of $\mathrm{CP}, \mathrm{nnHA}$ and $\mathrm{iHA}$ groups (E). Alizarin red staining of scaffolds $(\mathrm{n}=5)(\mathrm{F})$. Water contact angle illustrating increased hydrophilicity after hydroxyapatite coating ( $n=6-7)(G)$. 


\subsubsection{Crystal structure of surface and bulk modifications}

XRD analysis was further conducted to characterise the crystal structure between groups (Figure 2E). Brushite, hydroxylapatite and halite were identified in the pHA group, in close agreement with previous literature [51], with a crystallinity of $61.3 \%$. The $n n H A$ and iHA groups were both shown to be composed solely of hydroxyapatite, with crystallinity of $52.5 \%$ and $69.3 \%$ respectively. The broader peaks in the nnHA group are indicative of a smaller crystallite size, which was found to be $8.5 \mathrm{~nm}$. This is in comparison to the larger crystallite sizes in pHA and $\mathrm{iHA}$, which were measured at $105.8 \mathrm{~nm}$ and $75.0 \mathrm{~nm}$ respectively.

\subsubsection{Calcium content of $P C L$ modifications}

The total calcium content as determined via quantification of alizarin red staining was investigated following scaffold modification with calcium phosphate minerals (Figure 2F). The calcium detected in $C$ is minimal, and is due to slight background staining of the PCL. The greatest amount of calcium was found on pHA where nearly $150 \mu \mathrm{m}$ was detected and was significantly greater than all other approaches. nnHA contained the second largest quantities of calcium but was still 6-fold less than that with pHA and was not significantly different to iHA. The calcium content detected in iHA is only marginally greater than that detected from background staining $(C)$ due to the majority of $H A$ particles being distributed throughout the bulk of the fibres.

\subsubsection{Surface treatment enhances material hydrophilicity}

The hydrophilicity of groups was investigated and found to correlate closely with their calcium content (Figure 2G). This is seen in the complete spreading of water on pHA, which has the greatest calcium content. The nnHA group also exhibited a significant degree of hydrophilicity, with a mean contact angle of $56.0^{\circ}$. The $\mathrm{C}$ and $\mathrm{iHA}$ groups were both shown to be hydrophobic in comparison, with contact angles of $117.3^{\circ}$ and $117.1^{\circ}$ respectively.

\subsection{Mechanical properties are enhanced via HA-incorporation into PCL MEW fibres}

Incorporation of HA into PCL in iHA is seen to increase its stiffness and yield force, while all coating protocols have minimal influence on mechanical behaviour (Figure $3 A, B$ ). Stiffness in the linear elastic region between $0.1-0.3 \mathrm{~N}$ is greatest in iHA scaffolds (Figure $3 \mathrm{C}$ ), while stiffness in the toe region is not seen to be significantly altered between groups (Figure 3D). Yield force of iHA scaffolds is also significantly enhanced compared to all other groups, with an average fold increase of 1.4. 

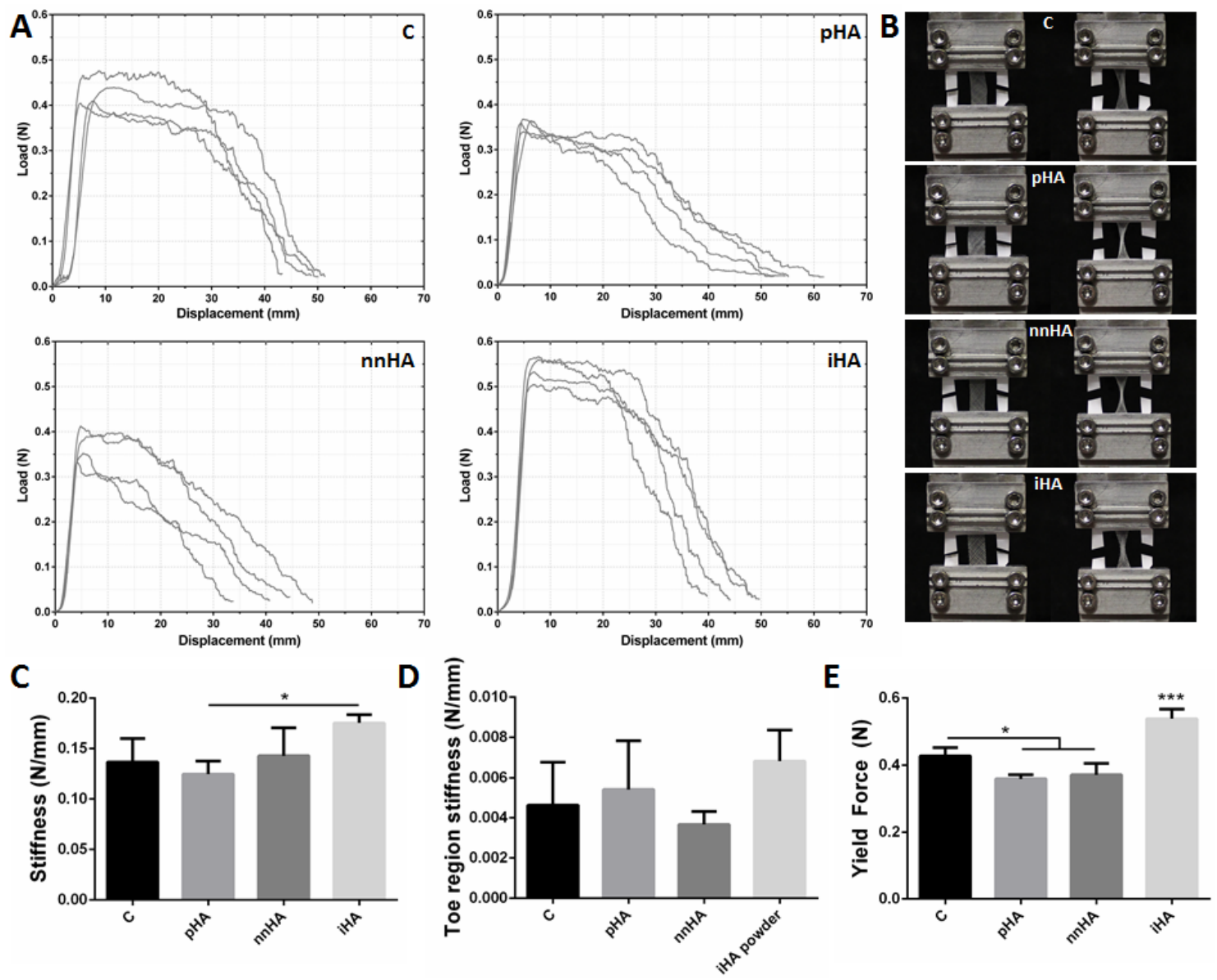

Figure 3 Mechanical characterisation of scaffolds via tensile testing. The incorporation of HA into fibres (iHA) can be seen to alter the mechanical properties of PCL micro-fibres (A). Scaffolds before tensile testing, and after being loaded to approximately $50 \%$ of the yield strain (B). Significant increases in stiffness seen in iHA (C), with no change in toe region stiffness (D). Yield force of iHA scaffolds is also significantly increased (E). (n=4).

\subsection{Attachment and proliferation of hMSCs}

Cells adhere to scaffolds with an average seeding efficiency of $25 \%$, with approximately $1,100-$ 3,400 cells remaining as quantified after $24 \mathrm{~h}$ post seeding. Adherent cells were found to branch across fibres resulting in elongated. Adherent hMSCs were commonly found branching across adjacent fibres as has been previously demonstrated with this fibre architecture [28]. Interestingly the pHA coating resulted in a slightly altered cellular morphology will hMSCS adhering and spreading across the deposited mineral, masking the underlying fibre architecture (Figure 4A). The initial cell seeding efficiency is lowest in the coated scaffold groups; pHA and nnHA. This trend is seen to level off by D7 however, where cell number reaches the initial seeding quantity at a number of 10,000 per scaffold. Cells then proliferate 2 -fold per week for the remainder of the study, with an average of 

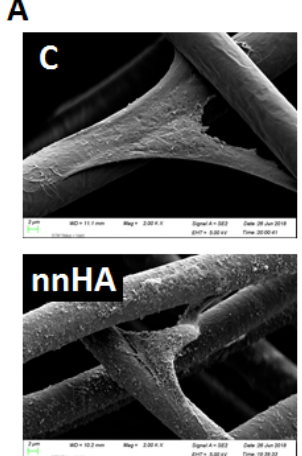
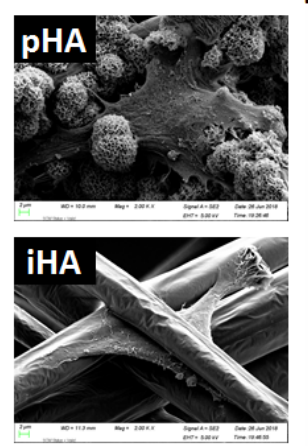

B

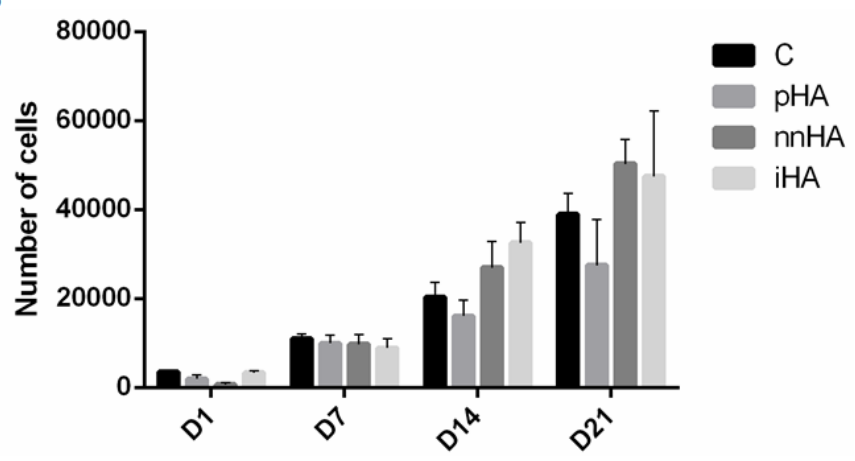

Figure 4 SEM imaging of cell seeded scaffolds after $24 \mathrm{~h}$ culture (A) Cell proliferation as quantified by DNA content.

Proliferation in pHA scaffolds can be seen to be attenuated D14 compared to other scaffold groups (B). ( $n=4)$.

\subsection{Surface treatment significantly enhances hMSC osteogenesis}

\subsubsection{ALP activity}

402 No significant trends were identified in hMSC ALP expression between each scaffold at D14 and D21.

403 Total ALP activity can however be seen to be greater in nnHA and iHA groups at these time-points 404 (Figure 5A). Of note is the 2.2-fold increase in ALP activity in nnHA at D21 compared to D14, in contrast with all other groups which remain constant between these time-points. Normalising these results to cell number reveals no further trends in ALP expression (Figure 5B), which remains relatively constant between groups.

A

408

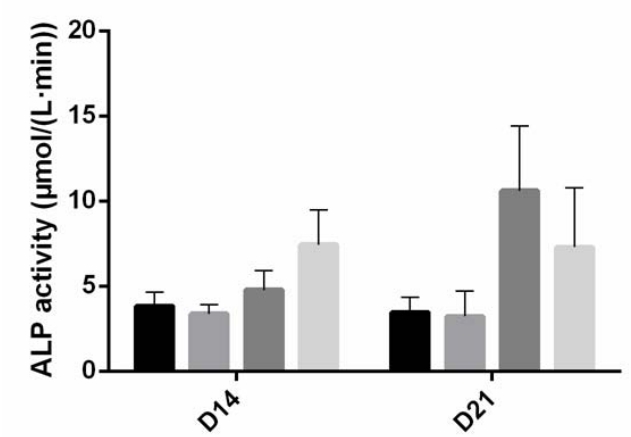

B

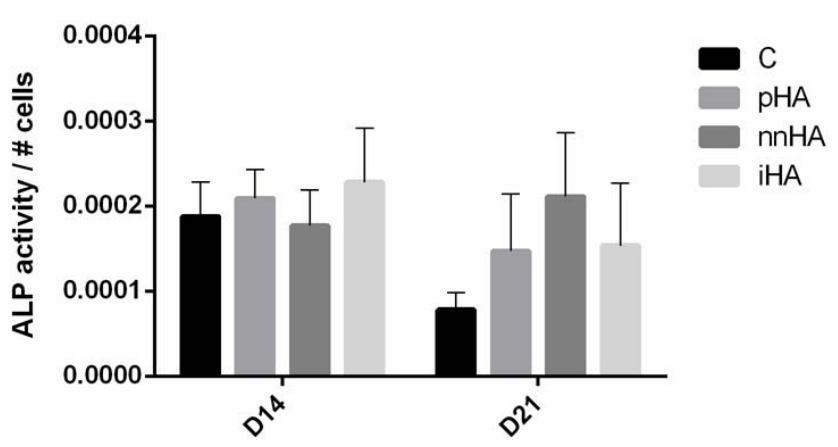


411

412

413

414

415

416

417

418

419

420

421

422

423

424

425

426

427

428

429

430

431

432

433

434

435

\subsection{2 hMSC Collagen production}

Collagen production is shown to be enhanced in coated scaffold groups (Figure 6). Total collagen quantity is greatest in pHA and nnHA at D21, with a 1.7 and 1.5-fold increase respectively compared to control scaffolds. This effect is further magnified in the pHA group when normalising results to cell number (Figure 6C), which is significantly greater than other groups. Collagen content per cell is 3.4fold greater in pHA than control, and over 2.5-fold greater than nnHA and iHA groups.

A

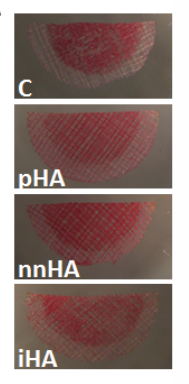

B

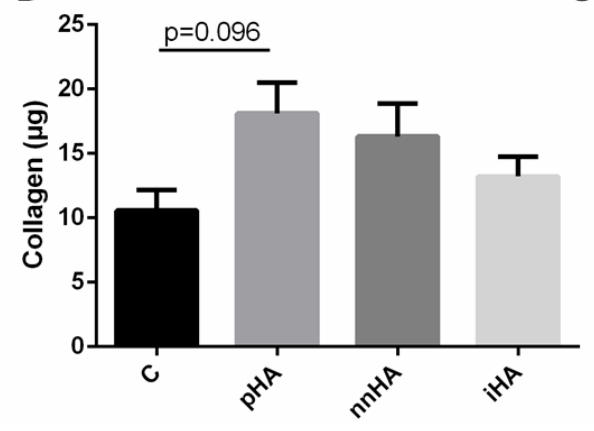

C

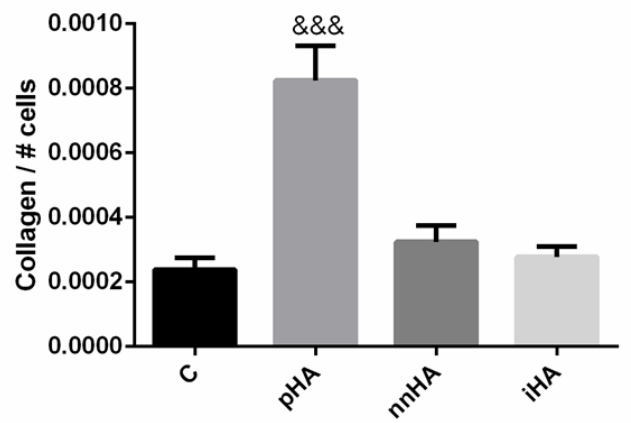

Figure 6 Collagen production at D21 (A). Total collagen is enhanced in the pHA group compared to C (A), while a significant increase compared to all groups is seen when collagen is normalised to cell number $(B) .(n=4)$. $\&=$ statistical significance compared to all other groups $(\& \& \&=p<0.001)$.

\subsection{3 hMSC Calcium production}

As with previous outputs of osteogenic differentiation, mineral production is seen to be enhanced in the coated scaffold groups (Figure 7A). These results encompass both mineralisation due to calcium production by cells, as well as the calcium present due to the treatments prior to cell seeding. Total mineral in pHA was 6.7-fold greater than $\mathrm{C}$ and iHA (Figure 7B). The greatest concentration following 21 days culture was found on scaffold nnHA, with this being over 12 -fold greater than the calcium content in $\mathrm{C}$ and $\mathrm{iHA}$ and 1.8 -fold greater than pHA. These findings are further enhanced when considering calcium due to cell mineralisation alone. The cell contribution to mineral in pHA was $59 \%$, with the remainder the mineral from the scaffold coating. In stark contrast, $92 \%$ of the mineral in nnHA is contributed by the cells. Considering this cell mineralisation alone, nnHA is over 14-fold greater than $\mathrm{C}$ and 2.9 -fold greater than $\mathrm{pHA}$. When total calcium is normalised to cell number at D21 (Figure 6C), calcium in pHA and nnHA groups are seen to be significantly greater than others, with an over 10-fold increase in mineral production per cell in pHA and over 12-fold increase in nnHA when compared to untreated control PCL scaffolds. 


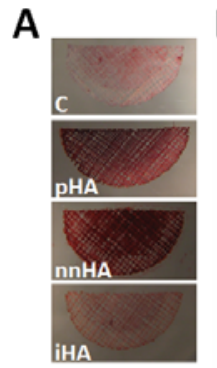

B

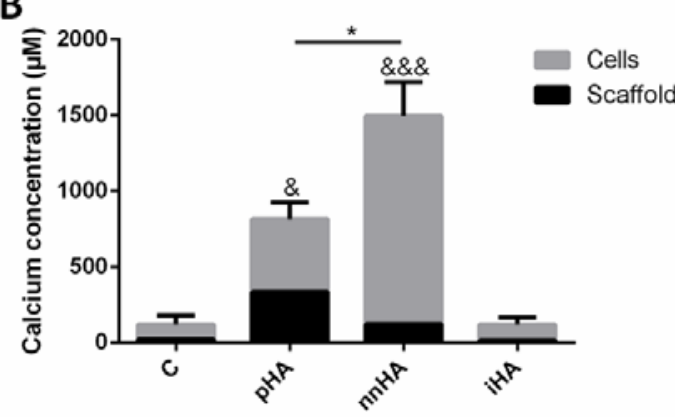

C

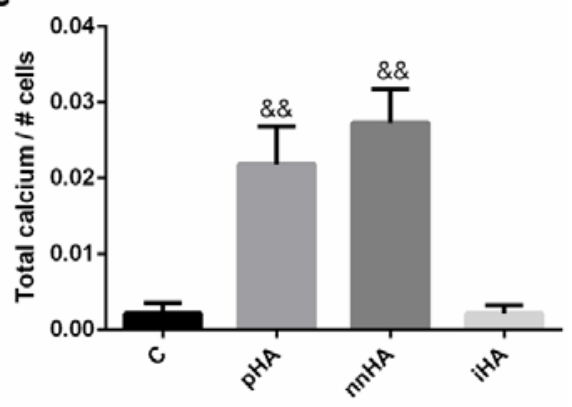

437 Figure 7 Calcium content at D21 with contributions from scaffold and cell mineralisation (A). Total calcium content is 438 significantly enhanced in nnHA and pHA groups (B). Calcium content normalsied to cell number is also enhanced in these 439 groups compared to $\mathrm{C}$ and $\mathrm{iHA}(\mathrm{C})$. $(n=4)$. \& = statistical significance compared to $\mathrm{C}$ and $\mathrm{iHA}$ groups $(\&=p<0.05, \& \&=$ $440 \quad p<0.01, \& \& \&=p<0.001){ }^{*}=p<0.05$.

\subsection{Coating dissolution after long term culture}

442 After 21 days in culture, the pHA coating was found to be largely intact as seen via SEM imaging, 443 with minor changes seen in a more rough surface topography (Figure 8B) compared to at D1 (Figure $4448 \mathrm{~A})$. In stark contrast to this, the nnHA coating is seen to have undergone considerable dissolution, 445 with loss of the nano-needle topography and formation of a coating of nano-spheres with diameter 446 of approximately 5-20 $\mathrm{nm}$. Consistent fracturing of this coating can also be seen. The iHA group is 447 also considerably altered, with extensive precipitates seen at the fibre surface in contrast to the 448 relatively smooth fibre topography at $\mathrm{D} 1$. 
A
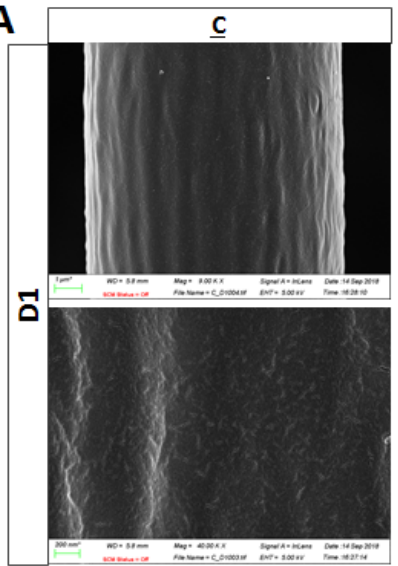

B

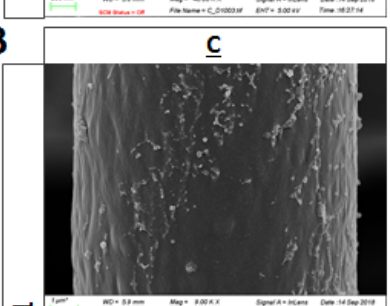

ลี

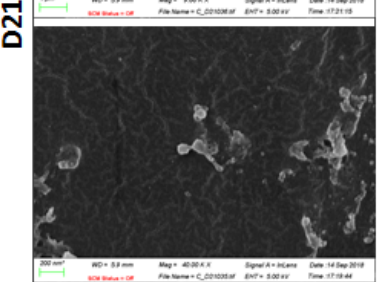

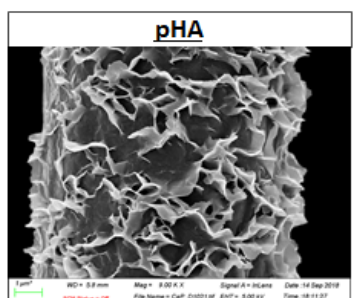

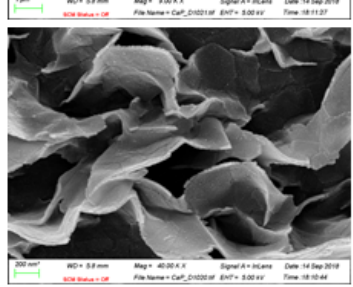

pHA
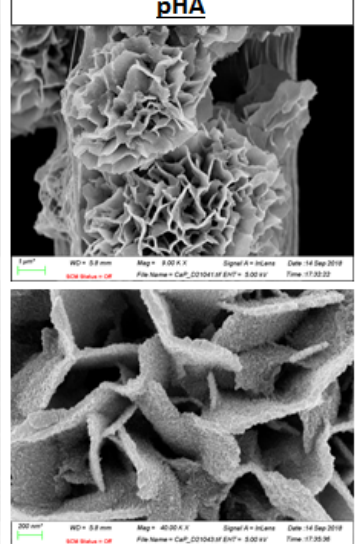
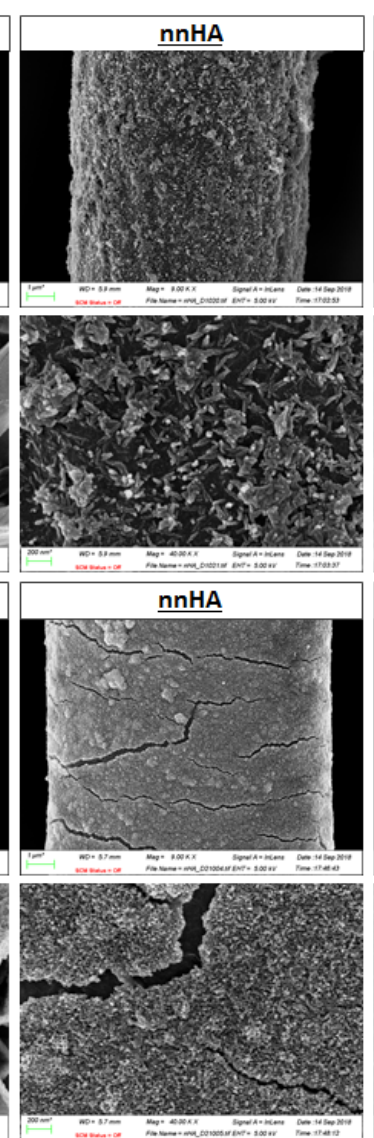
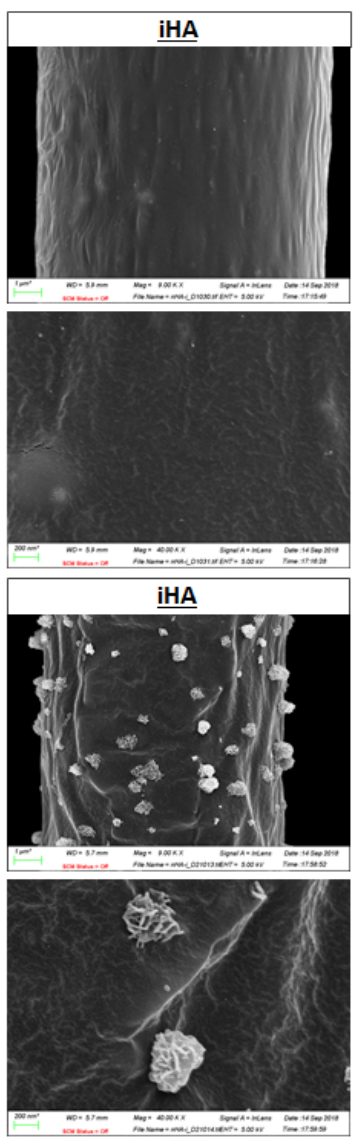

Figure 8 Fibre appearance after day 1 (A) and day 21 (B) in culture. Plates in the pHA group appear more rough at D21, with morphology otherwise unchanged. The nnHA coating is significantly altered, with the initial nano-needles becoming spherical and smaller in nature, and the coating exhibiting widespread fracturing. A greater amount of mineral precipitation on the fibre surface is seen in iHA at D21, where the near-surface HA appears to act as sites for mineral nucleation.

\subsection{Mineral architecture mediates BMP2 controlled release and further enhances hMSC} osteogenesis

In order to investigate the capacity of mineral architectures to bind, stabilise, and release growth factors, scaffolds were loaded with BMP2 and release kinetics and stem/stromal cell osteogenesis investigated. There is an initial burst release of BMP2 from the $\mathrm{C}, \mathrm{pHA}$ and $\mathrm{iHA}$ materials, with a more sustained initial controlled release from nnHA (Figure 9A). The majority of the BMP2 is released by $C$ after 7 days with a total amount of approximately $50 \%$, where the curve is seen to plateau with minimal further release up to day 21 , indicating a lower level of adsorption of the protein with reduced affinity to the scaffold (Figure 9B). At 7 days, release percent from pHA and iHA 
466 is $65 \%$ and $61 \%$ respectively, in contrast to $n n H A$, with a value of $46 \%$. Between D7 and D21, nnHA

467 continues to maintain the greatest level of controlled release at a rate of $9.8 \%$ per week.

468 The controlled release of BMP2 from the MEW scaffolds is seen to enhance cell proliferation in each

469 group. Of particular note is the significant 1.9-fold increase in cell number on the nnHA scaffolds at

470 day 21 compared to control nnHA scaffolds, in addition to significantly enhanced DNA in BMP2

471 treated nnHA vs pHA, with a fold change of 2.9 (Figure 9C). The effect of BMP2 delivery on the

472 osteogenic differentiation of hMSCs was investigated via ALP activity, collagen and mineral

473 deposition as before. ALP increases were only seen in the C scaffolds, however as with all other

474 groups, this was not significant (Figure 9D). Collagen content was also investigated, with the greatest

475 increases in C and iHA compared to control scaffolds (Figure 9E). Overall collagen content was

476 between 16-20 $\mathrm{g}$ in all groups, with greatest collagen content in nnHA after 21 days. Finally,

477 mineralisation is further enhanced in CP BMP2 functionalised scaffolds, with the greatest changes

478 seen in the nnHA and iHA groups, with increases of $259 \mu \mathrm{M}$ and $372 \mu \mathrm{M}$ respectively (Figure 9F). In

479 summary, the nnHA group exhibits the greatest potential for modification with additional factors, as

480 seen by the greatest level of sustained protein release, significantly enhanced DNA content, and the

481 greatest levels of collagen content and mineralisation content following 21 days in culture. 
A

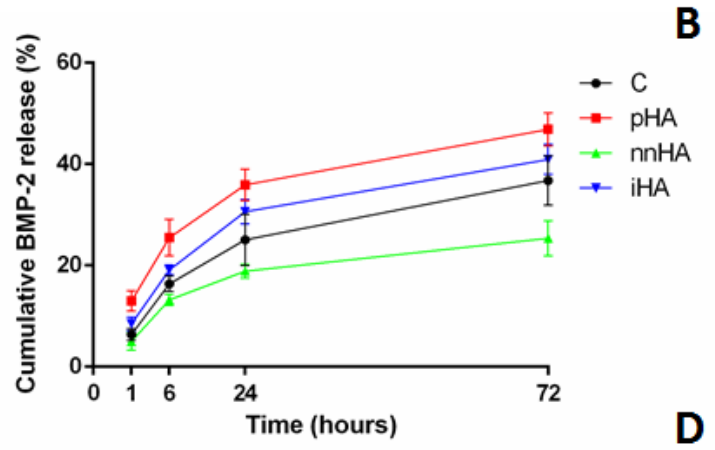

C
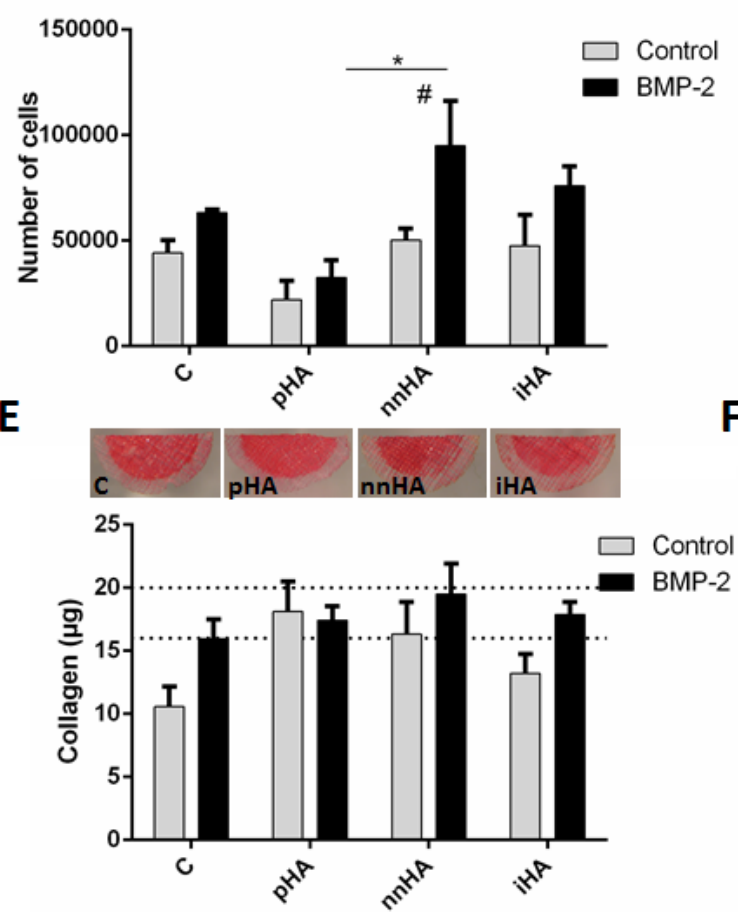

B

Control

BMP-2

$\mathbf{F}$
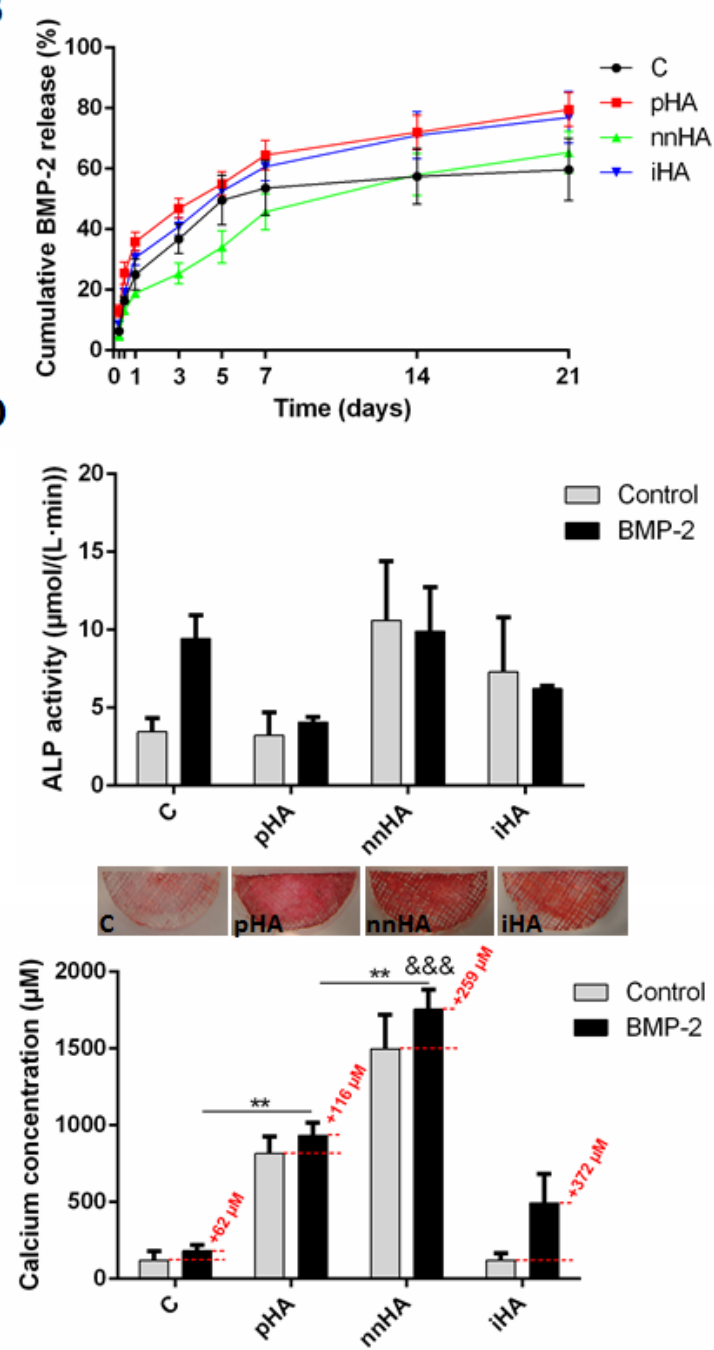

Figure 9 Cumulative BMP2 release up to D3 (A) and up to D21(B) ( $n=4)$. Number of cells (C), ALP activity (D), collagen content (E) and calcium content (F) with BMP2 treatment at D21 compared to data from untreated scaffolds as presented in previous figures. * = statistical significance via one-way ANOVA and Tukey multiple comparison test within BMP2 group only. \& = statistical significance vs $C$ and iHA groups with same method as previously. \# = statistical significance between Control and BMP2 in the nnHA group via two-way ANOVA and Bonferroni's multiple comparison test.

\section{Discussion}

490 Due to a lack of bone autograft supply, bioinspired synthetic alternative materials are required to enhance bone regeneration in critically sized defects. Cell behaviour and function are mediated by microenvironmental biophysical and biochemical cues, with the architecture and chemistry of the surrounding extracellular matrix being fundamental in this regard. Bone at a cellular level consists of arranged fibres with a cross-fibrillar mineral phase made up of curved nano-sized needle shaped 
495

496

497

498

499

500

501

502

503

504

505

506

507

508

509

510

511

512

513

514

515

516

517

518

519

520

521

522

523

524

525

526

527

528

crystals. Recapitulating this unique multiscale architecture may represent an innovative approach to develop effective bioinspired materials to promote bone regeneration. In this study, we utilise a $90^{\circ}$ fibrous MEW scaffold architecture, which we have previously optimised for human MSC osteogenesis [28], and built on this foundation by developing biomimetic mineral functionalisation strategies consisting of either intrafibrillar or cross fibrillar configurations. Interestingly using a novel coating approach, we were able to grow nano-needle shaped HA (nnHA) crystals on the surface of the MEW fibres which closely mimic that found in bone. These bioinspired nnHA topographies significantly enhanced hMSC osteogenesis over traditional SBF plate HA (pHA) coatings or intrafibrillar HA (iHA) scaffolds. Moreover, given the protein stabilising properties of mineral, we demonstrated that nnHA facilitated a controlled release of BMP2 from the scaffold which further enhanced mineral deposition by stem/stromal cells. This study thus outlines a method for fabricating scaffolds with precise fibrous micro-architectures and bone mimetic nano-needle HA extrafibrillar coatings which significantly enhance MSC osteogenesis and therapeutic delivery and thus hold great promise for bone tissue regeneration.

The microarchitecture/topography, material chemistry, and mechanical properties of each MEW scaffolds is altered followed CaP modifications. The concentrated SBF pHA coating technique resulted in the formation of plates arranged in a "cauliflower" or "rosette" like coating morphology, consistent with previously published findings using this approach [51-53] and typical of the plate or "petal-like" morphology of apatite formed via supersaturated solutions [54]. In contrast, the nnHA coating procedure which we have developed resulted in a vastly different morphology consisting of a fine, extrafibrillar, nano-needle coating which form occasional aggregates, closely mimicking the fundamental mineral units of bone in terms of morphology and composition recently identified [4]. The incorporation of $\mathrm{HA}$ into $\mathrm{PCL}$ in the $\mathrm{iHA}$ group does not have a significant influence on fibre surface morphology, as seen by SEM and water contact angle measurements, however, mechanical properties are enhanced with this approach. EDX analysis was further conducted to investigate the elemental distribution and composition of modified fibres. The $\mathrm{Ca} / \mathrm{P}$ ratios of all groups were within the range seen in native bone, which has typical $\mathrm{Ca} / \mathrm{P}$ ratios varying between $1.34-2.17$ with a median value of 1.69, as seen in samples from the femoral neck and the rib bones of healthy humans [55-57] (note: weight ratios in references were converted to atomic ratios). To further investigate the crystal structure of our scaffold groups, we performed an x-ray diffraction analysis. Interestingly, the broad peaks of the XRD spectra of nnHA closely mimic those of native bone. This is in comparison to the other groups which are more crystalline in nature. In summary, we have demonstrated that our nnHA coating procedure closely mimics the nano-architecture, composition and crystal structure of native bone. 
529 Bone bioinspired architectures can significantly enhance MSC mediated bone formation, as we have 530 demonstrated via long term in-vitro studies. Cell behaviour was investigated on modified scaffolds 531 via proliferation and markers of osteogenic differentiation. Cell number was greatest in the nnHA 532 group after 21 days, with the lowest number of cells in the pHA group. This is consistent with 533 previous work demonstrating a trend of increased proliferation with reduced HA particle size [34]. 534 We have also demonstrated a corresponding increase in ALP activity in nnHA, however, this result is 535 not significant. It is known that osteoprogenitor cells initially produce ALP and secrete collagen type 536 I, with further mineralisation occurring on this matrix at later time-points $[58,59]$. We have shown 537 previously that the fibrous architecture of scaffolds can have a significant influence on hMSC 538 collagen production [28]. However, our current findings indicate that HA surface topography does 539 not augment collagen production. Further investigation of calcium deposition demonstrated highly 540 significant upregulation, particularly with nnHA coating. The majority of the contribution towards 541 total calcium content is seen to be due to cell-based mineralisation, indicating the significant 542 influence of nano-scale needles in this extrafibrillar coating in driving stem/stromal cell osteogenic 543 differentiation. There are several likely factors for this result, in addition to the influence of the 544 nano-particle size as discussed previously. Greater dissolution of this coating, as demonstrated by 545 SEM imaging after 21 days in culture will yield a greater release of calcium and phosphate ions into 546 the medium which can interact with cells and allow for reprecipitation, enhancing mineralisation 547 [60]. In addition, an inverse relationship between crystallinity and mineralisation has also been 548 shown [61], providing further evidence for the greater cell mineralisation on our nnHA group, which 549 has the lowest crystallinity. This therefore suggests that the superior bone formation demonstrated 550 with the nnHA coating is multifactorial, harnessing several mechanisms to maximise regeneration.

551 Nano-needle structured HA extrafibrillar coatings effectively bind, and slowly deliver stable BMP2, 552 enhancing hMSC proliferation and bone formation. We investigated the protein binding and release 553 capacity of these scaffold modifications via a BMP2 adsorption study. Recent studies have 554 demonstrated that mineral can stabilise bound proteins, preventing conformational changes, 555 maintaining biological activity for weeks during release and that this stabilisation is enhanced within 556 nanostructured coatings [6]. The nnHA group was shown to facilitate the most sustained release of 557 BMP2 over 21 days. Interestingly, this resulted in significantly enhanced proliferation in this group 558 demonstrating biological activity, while markers of osteogenic differentiation were also marginally 559 increased. A previous study has reported similar results, where continuous supplementation of 560 media with BMP2 resulted in enhanced proliferation of hMSCS without initiating osteogenic 561 differentiation [62]. However, many studies have also demonstrated the key role of BMP2 in 562 stimulating osteogenesis [63-65]. We believe that due to the high degree of mineralisation indicating 
563 late stage differentiation, day 21 may be too late to capture elevated ALP expression, which is an

564 early differentiation marker. Consistent with previous findings in this paper, we also believe that

565 peak collagen levels for our scaffolds are being reached, as seen by the attenuated difference

566 between groups with BMP2 treatment compared to untreated scaffolds. While no significant

567 increases in calcium content were identified, it must be noted that the levels in nnHA are already 14-

568 fold greater than control scaffolds in the absence of BMP2 treatment, and the further increases in

569 mineral with BMP2 treatment in nnHA and iHA groups represent a change several fold-times greater

570 than basal calcium levels in untreated control scaffolds, which we have previously shown to

571 represent significantly enhanced mineralisation compared to random scaffolds [28]. In summary,

572 scaffolds with nnHA coating are shown to significantly enhance stem/stromal cell differentiation,

573 with the controlled release of BMP2 loaded scaffolds further contributing to this effect.

\section{Conclusions}

575 In conclusion, we utilised MEW technology to create fibrous 3D micro-architectures and further

576 modified these templates with a novel bioinspired extrafibrillar coating of needle shaped nano-sized

577 crystals (nnHA). This bone mimetic fibrous and mineral architecture significantly enhanced human

578 MSC osteogenesis over more established plate like mineral coatings. Moreover, extrafibrillar

579 coatings of nano-needles facilitated the binding, stabilisation, and controlled release of BMP2 from

580 the material which further enhanced MSC cell proliferation and bone formation. This study thus

581 outlines a method for fabricating scaffolds with precise fibrous micro-architectures and bone

582 mimetic nano-needle HA extrafibrillar coatings which significantly enhance MSC osteogenesis and

583 therapeutic delivery and thus hold great promise for bone tissue regeneration.

\section{Funding}

585 The authors would like to acknowledge funding from European Research Council (ERC) Starting

586 Grant (336882), Science Foundation Ireland (SFI) Support Grant SFI 13/ERC/L2864 and Irish Research

587 Council Postgraduate Scholarship (GOIPG/2014/493).

\section{$588 \quad 8$ References}

589 1. Yaszemski, M.J., et al., Evolution of bone transplantation: molecular, cellular and tissue 590 strategies to engineer human bone. Biomaterials, 1996. 17(2): p. 175-85. 
591 2. Winkler, T., et al., A review of biomaterials in bone defect healing, remaining shortcomings 592 and future opportunities for bone tissue engineering: The unsolved challenge. Bone Joint Res, $593 \quad$ 2018. 7(3): p. 232-243.

594 3. Tzaphlidou, M., Bone Architecture: Collagen Structure and Calcium/Phosphorus Maps. 595 Journal of Biological Physics, 2008. 34(1-2): p. 39-49.

5964 4. Reznikov, N., et al., Fractal-like hierarchical organization of bone begins at the nanoscale. $597 \quad$ Science, 2018. 360(6388).

598 5. Asara, J.M., et al., Protein sequences from mastodon and Tyrannosaurus rex revealed by mass spectrometry. Science, 2007. 316(5822): p. 280-5.

6. Yu, X., et al., Nanostructured Mineral Coatings Stabilize Proteins for Therapeutic Delivery. Adv Mater, 2017. 29(33).

7. Alford, A.I., K.M. Kozloff, and K.D. Hankenson, Extracellular matrix networks in bone remodeling. The International Journal of Biochemistry \& Cell Biology, 2015. 65: p. 20-31.

9. Kane, R. and P.X. Ma, Mimicking the nanostructure of bone matrix to regenerate bone. Materials Today, 2013. 16(11): p. 418-423.

10. Lu, P., et al., Extracellular Matrix Degradation and Remodeling in Development and Disease.

11. Bonnans, C., J. Chou, and Z. Werb, Remodelling the extracellular matrix in development and

12. Hutmacher, D.W., et al., Mechanical properties and cell cultural response of

13. Di Luca, A., et al., Tuning Cell Differentiation into a 3D Scaffold Presenting a Pore Shape

14. Daly, A.C., et al., 3D Bioprinting for Cartilage and Osteochondral Tissue Engineering. Advanced Healthcare Materials, 2017. 6(22): p. 1700298.

15. Cox, S.C., et al., 3D printing of porous hydroxyapatite scaffolds intended for use in bone tissue engineering applications. Materials Science and Engineering: C, 2015. 47: p. 237-247.

16. Fierz, F.C., et al., The morphology of anisotropic 3D-printed hydroxyapatite scaffolds. Biomaterials, 2008. 29(28): p. 3799-3806. 
625 17. Bergmann, C., et al., 3D printing of bone substitute implants using calcium phosphate and 626 bioactive glasses. Journal of the European Ceramic Society, 2010. 30(12): p. 2563-2567.

627 18. Inzana, J.A., et al., 3D printing of composite calcium phosphate and collagen scaffolds for 628 bone regeneration. Biomaterials, 2014. 35(13): p. 4026-4034.

20. Labour, M.-N., et al., Electrospun Poly-D-L-Lactic Acid Fibrous Scaffolds as a Delivery Vehicle

21. Zhou, P., et al., Loading BMP-2 on nanostructured hydroxyapatite microspheres for rapid

22. Brown, T.D., P.D. Dalton, and D.W. Hutmacher, Direct writing by way of melt electrospinning. Adv Mater, 2011. 23(47): p. 5651-7.

23. Hochleitner, G., et al., Additive manufacturing of scaffolds with sub-micron filaments via melt electrospinning writing. Biofabrication, 2015. 7(3).

24. Wunner, F.M., et al., Melt Electrospinning Writing of Three-dimensional Poly(epsilon-
caprolactone) Scaffolds with Controllable Morphologies for Tissue Engineering Applications. J

26. Castilho, M., et al., Melt Electrowriting Allows Tailored Microstructural and Mechanical

27. Hochleitner, G., et al., Melt electrowriting below the critical translation speed to fabricate

28. Eichholz, K.F. and D.A. Hoey, Mediating human stem cell behaviour via defined fibrous international : a journal established as result of cooperation between the European 
658 30. Boskey, A.L., Bone composition: relationship to bone fragility and antiosteoporotic drug effects. BoneKEy Reports, 2013. 2: p. 447.

31. Habraken, W., et al., Calcium phosphates in biomedical applications: materials for the future? Materials Today, 2016. 19(2): p. 69-87.

32. LC, C., Next generation calcium phosphate-based biomaterials. Dental materials journal, 2009. 28(1): p. 1-10.

33. Zhou, H. and J. Lee, Nanoscale hydroxyapatite particles for bone tissue engineering. Acta Biomaterialia, 2011.7(7): p. 2769-2781.

34. Cai, Y., et al., Role of hydroxyapatite nanoparticle size in bone cell proliferation. Journal of Materials Chemistry, 2007. 17(36): p. 3780-3787.

35. Yang, X., et al., In Vitro Uptake of Hydroxyapatite Nanoparticles and Their Effect on

36. Remya, N.S., et al., An in vitro study on the interaction of hydroxyapatite nanoparticles and

37. Liu, Y., et al., In vitro effects of nanophase hydroxyapatite particles on proliferation and

38. Domingos, M., et al., Three-dimensional printed bone scaffolds: The role of nano/microhydroxyapatite particles on the adhesion and differentiation of human mesenchymal stem cells. Proc Inst Mech Eng H, 2016: p. 954411916680236.

40. Duan, R., et al., Accelerated bone formation by biphasic calcium phosphate with a novel sub-

41. Xiong, L., et al., BMP2-loaded hollow hydroxyapatite microspheres exhibit enhanced osteoinduction and osteogenicity in large bone defects. International journal of nanomedicine, 2015. 10: p. 517-526. scaffolds with and without tailored microporosity to deliver bone morphogenetic protein 2. Journal of Biomedical Materials Research Part A, 2005. 76A(2): p. 366-376. 
691 43. Notodihardjo, F.Z., et al., Bone regeneration with BMP-2 and hydroxyapatite in critical-size calvarial defects in rats. Journal of Cranio-Maxillofacial Surgery, 2012. 40(3): p. 287-291.

45. Curtin, C.M., et al., Innovative Collagen Nano-Hydroxyapatite Scaffolds Offer a Highly

46. Palazzo, B., et al., Biomimetic Hydroxyapatite-Drug Nanocrystals as Potential Bone

47. Sun, W., et al., Biodegradable Drug-Loaded Hydroxyapatite Nanotherapeutic Agent for

48. Martine, L.C., et al., Engineering a humanized bone organ model in mice to study bone

56. Zaichick, V. and M. Tzaphlidou, Calcium and phosphorus concentrations and the

49. Kumta, P.N., et al., Nanostructured calcium phosphates for biomedical applications: novel synthesis and characterization. Acta Biomaterialia, 2005. 1(1): p. 65-83.

50. Cunniffe, G.M., et al., The synthesis and characterization of nanophase hydroxyapatite using a novel dispersant-aided precipitation method. J Biomed Mater Res A, 2010. 95(4): p. 1142-9.

51. Vaquette, C., et al., Effect of culture conditions and calcium phosphate coating on ectopic bone formation. Biomaterials, 2013. 34(22): p. 5538-51.

52. Tas, A.C. and S.B. Bhaduri, Rapid coating of Ti6Al4V at room temperature with a calcium phosphate solution similar to 10x simulated body fluid. Journal of Materials Research, 2011. 19(9): p. 2742-2749.

53. Kokubo, T., et al., Solutions able to reproduce in vivo surface-structure changes in bioactive glass-ceramic A-W3. Journal of Biomedical Materials Research, 1990. 24(6): p. 721-734.

54. Drouet, C., Apatite Formation: Why It May Not Work as Planned, and How to Conclusively Identify Apatite Compounds. BioMed Research International, 2013. 2013: p. 490946.

55. Zaichick, V. and M. Tzaphlidou, Determination of calcium, phosphorus, and the calcium/phosphorus ratio in cortical bone from the human femoral neck by neutron activation analysis. Appl Radiat Isot, 2002. 56(6): p. 781-6.

calcium/phosphorus ratio in trabecular bone from the femoral neck of healthy humans as 
725

726

727

728

729

730

731

732

733

734

735

736

737

738

739

740

741

742

743

744

745

746

747

748

749

determined by neutron activation analysis. Applied Radiation and Isotopes, 2003. 58(6): p. 623-627.

57. Tzaphlidou, M. and V. Zaichick, Calcium, phosphorus, calcium-phosphorus ratio in rib bone of healthy humans. Biol Trace Elem Res, 2003. 93(1-3): p. 63-74.

58. Huang, Z., et al., The sequential expression profiles of growth factors from osteoprogenitors [correction of osteroprogenitors] to osteoblasts in vitro. Tissue Eng, 2007. 13(9): p. 2311-20.

59. Birmingham, E., et al., Osteogenic differentiation of mesenchymal stem cells is regulated by osteocyte and osteoblast cells in a simplified bone niche. Eur Cell Mater, 2012. 23: p. 13-27.

60. Chang, Y.-L., C.M. Stanford, and J.C. Keller, Calcium and phosphate supplementation promotes bone cell mineralization: Implications for hydroxyapatite (HA)-enhanced bone formation. Journal of Biomedical Materials Research, 2000. 52(2): p. 270-278.

61. Morgan, J., et al., In vitro mineralization and implant calcium phosphate-hydroxyapatite crystallinity. Implant Dent, 1996. 5(4): p. 264-71.

62. Lysdahl, H., et al., Preconditioning Human Mesenchymal Stem Cells with a Low Concentration of BMP2 Stimulates Proliferation and Osteogenic Differentiation In Vitro. BioResearch Open Access, 2014. 3(6): p. 278-285.

63. Beederman, M., et al., BMP signaling in mesenchymal stem cell differentiation and bone formation. Journal of biomedical science and engineering, 2013. 6(8A): p. 32-52.

64. Huang, W., et al., BMP-2 exerts differential effects on differentiation of rabbit bone marrow stromal cells grown in two-dimensional and three-dimensional systems and is required for in vitro bone formation in a PLGA scaffold. Experimental Cell Research, 2004. 299(2): p. 325334.

65. Li, C., et al., Electrospun silk-BMP-2 scaffolds for bone tissue engineering. Biomaterials, 2006. 27(16): p. 3115-3124. 